\title{
ANÁLISE DE PRIORIDADES DE MELHORIA EM RELAÇÃO À SATISFAÇÃO DOS CLIENTES DE UM MERCADO POR MEIO DE DOIS MODELOS DE MENSURAÇÃO
}

\author{
IMPROVEMENT PRIORITY ANALYSIS IN RELATION TO CUSTOMER SATISFACTION \\ OF A MARKET THROUGH TWO MEASUREMENT MODELS
}
Recebido em 17.11.2019 Aprovado em 10.12.2019
Avaliado pelo sistema double blind review DOI: https://doi.org/10.32888/cge.v7i3.38713

\section{Milena Fernanda Bogler Tavares}

milenabogler@gmail.com

Universidade Estadual do Oeste do Paraná - Marechal Candido Rondon/ Paraná/ Brasil.

\section{Silvana Anita Walter}

silvanaanita.walter@gmail.com

Universidade Estadual do Oeste do Paraná - Marechal Cândido Rondon/ Paraná/ Brasil.

\section{Mario Luiz Soares}

mario.soares@unioeste.br

Universidade Estadual do Oeste do Paraná - Marechal Cândido Rondon/ Paraná/ Brasil.

\section{Cinara Kottwiz Manzano Brenzan}

cinaramanzano@yahoo.com.br

Universidade Estadual do Oeste do Paraná - Marechal Cândido Rondon/ Paraná/ Brasil.

\section{Resumo}

Este artigo tem por objetivo analisar as prioridades de melhoria em relação à satisfação dos clientes de um mercado através do uso de dois modelos de mensuração: o Modelo Kano e a Matriz de Importância x Desempenho observando as diferenças entre eles, objetivando considerar alguns pontos da empresa que podem ser explorados como diferenciais para elevar a satisfação dos clientes. Para tanto, 18 atributos foram analisados de forma quantitativa, com uma amostra de 185 clientes. Alguns atributos prioritários para melhoria foram identificados no mercado, como variedades de produtos, promoções e qualidade dos produtos do açougue. Os resultados demonstram que a utilização conjunta dos modelos proporcionou maior qualidade na análise dos dados para a tomada de decisão.

Palavras-chave: Satisfação de Clientes. Matriz de Importância x Desempenho. Modelo Kano.

\section{Abstract}

The objective is to analyze the improvement priorities in relation to customer satisfaction of a market through the use of two measurement models: the Kano Model and the Importance $x$ Performance Matrix observing the differences between them, aiming to consider some points. that can be exploited as differentials to increase customer satisfaction. To this end, 18 attributes were analyzed quantitatively, with a sample of 185 clients. Some priority attributes for improvement have been identified in the market, such as product varieties, promotions and butcher product quality. The results show that the joint use of the models provided higher quality in the data analysis for decision making.

Keywords: Customer satisfaction. Importance x performance matrix. Kano model. 


\section{Introdução}

O processo de globalização e os avanços tecnológicos trazem como consequência o aumento da concorrência de mercado, da circulação de pessoas e bens, do incremento nos níveis dos sistemas de comunicação e informação. Isso leva as organizações a dobrarem a atenção com os clientes, que elevam seu nível de exigência, tornando as empresas cada vez mais competitivas e focadas no atendimento, que, associado ao conhecimento de mercado, será fundamental na obtenção da satisfação dos clientes (SILVA; SARAIVA, 2012; TRIERWEILLE; PRUDÊNCIO et al., 2011; AMARAL; DANTAS, 2015).

De modo geral, o varejo é tido como o estágio final da distribuição de bens e serviços, que engloba todas as atividades ligadas à venda ao consumidor. Para tanto, o varejista deve contar com a maior gama de variedade de produtos que puder desde que satisfaça o mercado - alvo em questão (SILVA, 2009).

$\mathrm{Na}$ busca pela satisfação dos clientes, é necessário prezar pela qualidade e pelo bom atendimento, sendo que, além de atender suas necessidades e/ou expectativas, deve-se estabelecer um bom relacionamento entre cliente e organização (COSTA et al., 2015). No varejo supermercadista isso não se difere, porém, necessita de atenção redobrada devido à crescente competitividade neste setor em especifico, sendo que obteve um acúmulo no aumento de vendas em 3,22\% recentemente (ABRAS, 2019). Portanto, focar no consumidor e se preocupar com a satisfação dos mesmos, são considerados critérios estratégicos e de extrema importância para manter a organização em funcionamento ao longo do tempo (OLIVEIRA, et al, 2013).

Ainda, é correto afirmar que o grau de satisfação dos clientes de uma empresa é decorrente de uma relação entre a perspectiva de um produto e/ou serviço e a visão posterior à aquisição do mesmo (MAGALDI; CRESCITELLI, 2008). Após essa aquisição, o cliente avalia o produto e/ou serviço de acordo com suas expectativas, podendo ficar satisfeito, insatisfeito ou extremamente satisfeito (KOTLER; ARMSTRONG, 2003), lembrando que um cliente satisfeito pode funcionar como um meio positivo de ampliação da carteira de clientes da organização fazendo uma espécie de "propaganda gratuita" para a mesma (SOUSA, 2011).

No entanto, se o cliente não se sentir satisfeito, poderá deixar de consumir determinado produto ou serviço, além de influenciar outros consumidores a fazerem o mesmo, transmitindo uma imagem negativa da empresa (MOUTELLA, 2003).

Esse tipo de pesquisa voltada à satisfação do cliente ganhou espaço nas empresas, pois possibilita ter uma base de quantos clientes estão satisfeitos ou não com os produtos e serviços ofertados por elas, buscando obter informações e melhorar continuamente. Os resultados dessas pesquisas minimizam as falhas e os erros apresentados, sendo fundamentais para um bom relacionamento com o públicoalvo (KOTLER, 2000; MARCHETTI; PRADO, 2004; SACHS, 2015; JURAN; DEFEO, 2015).

$\mathrm{Na}$ presente pesquisa, para a análise de dados, utilizou-se a Matriz de Importância x Desempenho, que determina os itens de menor desempenho, possibilitando identificar quais requisitos do cliente devem ser priorizados no plano de melhoria da empresa (FERNANDES, 2012). De modo complementar, o Modelo Kano identifica a reação ou a satisfação dos clientes perante os mesmos itens, quando perguntados se eles tivessem desempenho superior (positivo) ou inferior (negativa) do que têm atualmente (KANO et al., 1984; BERGER et al., 1933).

A presente pesquisa justifica-se através da apresentação de resultados reais e de valor para a empresa, além de gerar uma pequena experiência de consultoria e gestão ao acadêmico.

Buscando precisão nos resultados, têm-se como pergunta central deste artigo quais as prioridades de melhoria em relação à satisfação dos clientes do Mercado União?

Diante disso, o objetivo geral é analisar oportunidades de melhoria em relação à satisfação dos clientes por meio de dois métodos de mensuração. Já os objetivos específicos dizem respeito a: a) 
identificar o perfil dos respondentes; b) constatar a satisfação geral; c) apresentar discussões e análises firmadas pelos métodos de pesquisa; d) evidenciar os pontos fortes e fracos e, posteriormente, as sugestões de melhoria.

O artigo está estruturado em cinco seções: na próxima, apresenta-se o referencial teórico sobre satisfação de clientes, Matriz de Importância x Desempenho e Modelo Kano. Na terceira seção, descreveram-se os procedimentos metodológicos empregados para o desenvolvimento do estudo. $\mathrm{Na}$ quarta, tem-se a análise dos dados obtidos, descrevendo o caso e a análise de satisfação ou insatisfação dos clientes do mercado. Por fim, na quinta seção, estão as considerações finais que conclui o estudo.

\section{Referencial teórico}

Nesta seção, será apresentado o referencial teórico, que objetiva relatar a pesquisa empírica sobre satisfação dos clientes, Matriz de Importância x Desempenho e Modelo Kano de Qualidade Atrativa e Obrigatória, abordando conceitos, características e aplicabilidade.

\section{Satisfação de clientes}

A satisfação é vista de uma maneira consensual como uma das chaves para o sucesso das organizações (SOUSA, 2011; ALVES et al., 2015). A sobrevivência e a lucratividade da organização dependem diretamente da satisfação dos clientes, por isso, é importante, além de conhecer suas necessidades e seus desejos, superar suas expectativas no que for oferecido (DE JESUS et al., 2019). Assim, a satisfação tem um carácter relativo, isto é, a avaliação provém de um processo comparativo entre a experiência subjetiva vivida pelo cliente e um padrão de referência inicial, comparados com as expectativas e as percepções do desempenho do produto/serviço (SALOMÃO et al., 2018).

O consumidor vem elevando seu nível de exigência perante produtos e serviços, consequentemente, tornando as organizações mais competitivas, buscando diferenciais para obtenção da satisfação de seus clientes. Para tanto, o comportamento do consumidor se define como as atividades físicas e mentais que clientes de bens e serviços realizam e que resultam em decisões e ações. Da mesma forma, quando procuram por determinado produto, compram-no, utilizam-no, descartam-no e, por fim, avaliam-no, esperando que sacie suas necessidades e expectativas sobre ele (SHETH et al., 2001; BINOTTO et al., 2014; KANUK; SCHIFFMAN, 2009; SOLOMON, 2011).

Um consumidor em posição de escolher entre comprar ou não comprar ou de escolher entre a marca X ou Y está em uma posição de tomar uma decisão, ou seja, para que uma decisão ocorra, são necessárias alternativas distintas para que, posteriormente, haja escolha (KANUK; SCHIFFMAN, 2009).

Vários fatores podem ter influência durante o processo de tomada de decisão, sejam eles culturais, sociais, pessoais, psicológicos e até mesmo o desejo de compra do consumidor (KOTLER, 2006). Os fatores culturais incluem os costumes, a moral e os conhecimentos adquiridos pelo indivíduo em sociedade. Já nos sociais são estão os grupos de referência, a família e as posições e os papéis sociais. Os pessoais dizem respeito às particularidades de cada um (idade, ocupação, condições econômicas, estilo de vida e personalidade). E, nos psicológicos, existem cinco fatores importantes: a motivação, a percepção, a aprendizagem e as crenças e atitudes. Todos eles acabam por influenciar o consumidor (TARIGO et al., 2016).

Uma metodologia utilizada pela organização buscando definir produtos/serviços conforme a demanda dos clientes é o marketing, empregando estratégias nas vendas, agregando valor e possibilitando lucratividade para ambos os envolvidos (VIEIRA, 2018). Em uma era competitiva, o marketing de relacionamento, que advém do marketing, ganha destaque, baseando-se na interação entre clientes e colaboradores. É de interesse de ambos, já que o cliente busca por bom atendimento, descontos e qualidade nos produtos e serviços, e os colaboradores se empenham em 
atender esses requisitos e buscam satisfazer as necessidades dos consumidores, visando à frequência nas compras atreladas ao bom relacionamento (PAULA; SOUZA, 2016; SALIBY, 1997). Dessa forma, as organizações buscam fazer do relacionamento a principal arma para sobreviverem no mercado competitivo (COBRA, 2009).

No contexto atual, a satisfação dos clientes é de suma importância para que se tenha uma vantagem competitiva em relação aos concorrentes, classificando-a como item indispensável para a organização. $\mathrm{Na}$ análise da satisfação dos clientes em relação às suas expectativas sobre os produtos e/ou serviços desta organização, foram utilizados de forma conjunta dois modelos de mensuração: a Matriz de Importância x Desempenho e o Modelo Kano.

\section{Matriz de Importância x Desempenho}

A análise de Matriz Importância x Desempenho, originalmente introduzida por Martilla e James (1997), permite que a empresa tenha uma visão expressiva sobre quais atributos ligados a produtos ou serviços devem ser melhorados, proporcionando a satisfação dos clientes (WALTER, 2006). Além disso, baseia-se na importância de tais atributos e no desempenho que a empresa possui em relação à concorrência (RIGONI, 2010).

Geralmente, os dados provenientes de pesquisas de satisfação se dão por um questionário tipo Likert, que proporciona clareza na visualização a partir do posicionamento dos atributos nele contidos, que serão representados nos quadrantes do gráfico, revelando quais atributos devem ser melhorados, mantidos, ignorados ou estão superando as expectativas de desempenho (CUMMINS; GULLONE, 2000). Estes mesmos dados são utilizados para construção de uma matriz bidimensional, onde a importância do atributo é representada no eixo y e o desempenho do mesmo atributo pelo eixo x, conforme a Figura 1 (MATZLER et al., 2004; FIGUEREDO, 2005).

Sendo assim, os consumidores avaliam a real importância e o desempenho da empresa perante o atendimento de suas expectativas.

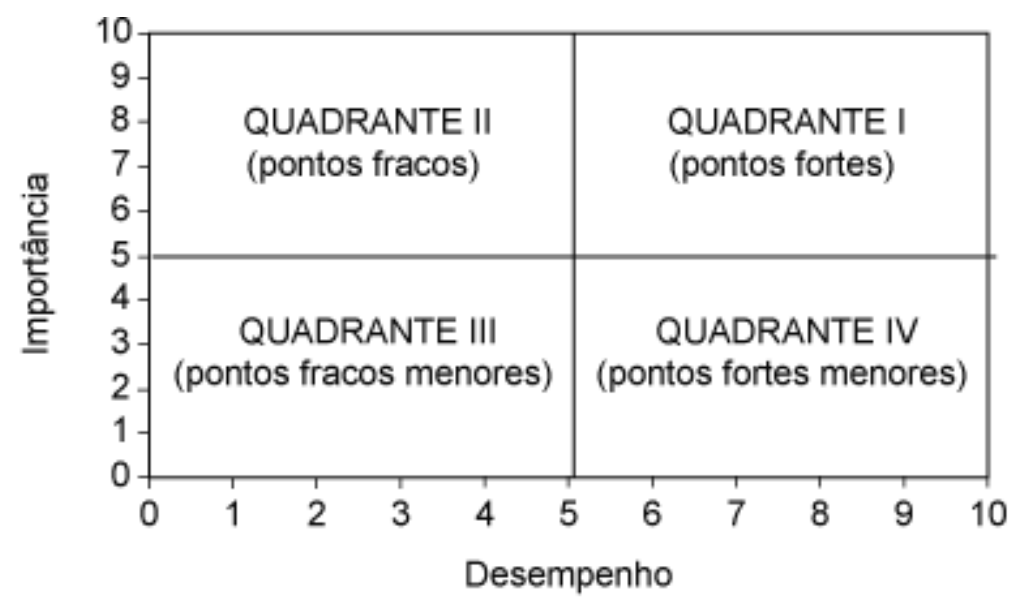

Figura 1 - Matriz de Importância x Desempenho.

Fonte: Adaptado de "The asymmetric relationship between attribute-level performance and overall customer satisfaction: a reconsideration of the importance-performance analysis" de Matzler, K; Bailom, F; Hinterhuber, H, H; Renzl, B; Pichler, J. V, 2004. Industrial marketing Management p. 271277.

A matriz é dividida em quatro quadrantes, como apresentado na Figura 1: o Quadrante I é considerado como um ponto forte e tem alta importância e alto desempenho, que representa uma possível vantagem competitiva. Portanto, a empresa deve manter o bom desempenho. No quadrante II, encontram-se os pontos fracos, ou seja, são atributos de alta importância, mas com desempenho 
abaixo da expectativa; então, deverão receber atenção imediata. O Quadrante III detém os atributos com baixa importância e baixo desempenho. É dispensável esforço complementar nesses atributos, que podem ser chamados de pontos fracos menores. Por fim, no Quadrante IV, concentram-se os atributos com alto desempenho, porém com baixa importância, e podem ser definidos pontos fortes menores. Para aumentar a satisfação geral, a empresa deve focar em melhorar os atributos nesse quadrante (TONTINI et al., 2004).

Porém, vale lembrar que o aumento no desempenho de um atributo de qualidade nem sempre condiz com o aumento da satisfação. Alguns atributos têm maior importância do que outros, e a importância de alguns se difere entre seguimentos de mercado ou entre os próprios clientes (MATZLER et al., 2008; SAUERWEIN et al., 1996).

Para complementar o estudo e trazer maior credibilidade a ele, a utilização do Modelo Kano trará a classificação dos atributos anteriormente elencados.

\section{Modelo Kano}

O Modelo Kano de Qualidade Atrativa e Obrigatória (KANO et al., 1984; BERGER et al., 1993; MATZLER et al., 1996) faz distinção entre três tipos de atributos ligados aos produtos ou serviços que influenciam a satisfação do cliente, sendo eles: obrigatórios, unidimensionais e atrativos (Figura 2).

Assim, os atributos obrigatórios referem-se às funções básicas de um produto. Se essas não estiverem presentes ou tiverem desempenho insuficiente, os clientes ficaram muito insatisfeitos. Os clientes veem estes atributos como pré-requisitos e, portanto, se eles não estiverem presentes ou forem suficientes, não trarão satisfação. Nos atributos unidimensionais, a satisfação do cliente é proporcional ao desempenho, ou seja, o aumento no grau de desempenho aumenta a satisfação do cliente, e vice-versa. Geralmente, esses atributos são exigidos com clareza pelos clientes.

Os atributos atrativos são pontos cruciais na busca pela satisfação dos clientes. Podem ser considerados como um ponto diferencial, uma vez que obter sucesso nesses atributos trará uma satisfação maior ao cliente. Por outro lado, se não houver sucesso nesses atributos, não causará insatisfação, já que são atributos não expressos e não são esperados pelo cliente (KANO et al., 1984).

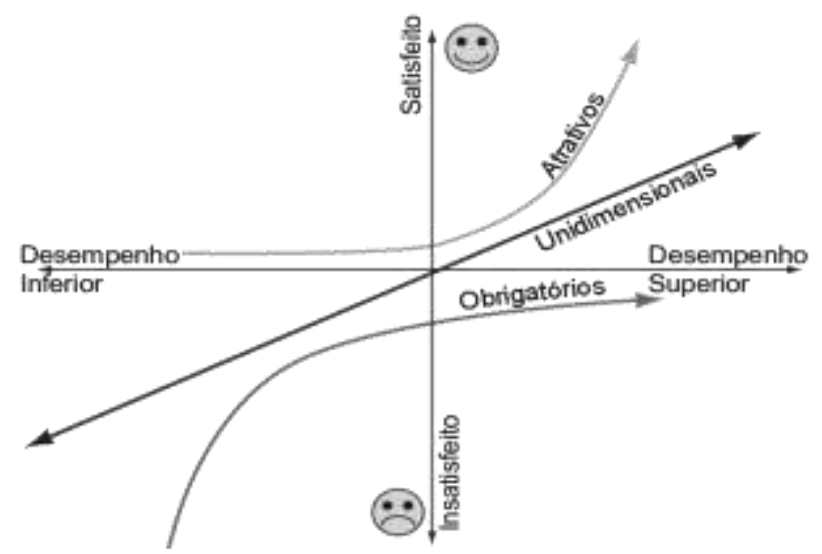

Figura 2 - Modelo Kano de Qualidade Atrativa e Obrigatória.

Fonte: Adaptado de "How to delight your customers". Matzler, K; Hinterhuber, H; Bailom, F; Sauerwein, E, 1996. Journal of Product \& Brand Management v. 5, 2, p. 6-18.

Além destes três atributos já citados por Kano et al., (1984), podemos identificar mais dois tipos: os neutros e os reversos. Os neutros são aqueles cuja presença não traz satisfação e sua ausência não traz insatisfação. São atributos que nunca, ou raramente, são usados pelo cliente ou, às vezes, o cliente não sabe como utilizá-los. E os atributos reversos são os quais sua presença traz insatisfação ao cliente (TONTINI, 2003).

Desta forma, fazendo uso dos dois métodos descritos, será possível uma melhor e mais eficiente 
análise dos dados coletados e consequentemente, uma melhor tomada de decisão perante as informações.

\section{Metodologia}

$\mathrm{Na}$ construção desta pesquisa, utilizou-se do método quantitativo, que significa traduzir em números as opiniões e as informações para classificá-las e analisá-las (GIL, 2002) com uma pesquisa descritiva e de levantamento. A pesquisa descritiva apresenta características de uma população, de um fenômeno ou estabelece relação entre as variáveis (GIL, 1999), buscando identificar, relatar e comparar (RAUPP; BEUREN, 2006) e a de levantamento obtém dados de um grupo de pessoas, que representam uma população (MEDEIROS, 2019).

No que tange a amostra da pesquisa, a população é de 300 clientes cadastrados, que fazem compras a prazo frequentemente. Os demais, que compram somente à vista, não possuem cadastro. Para o cálculo do tamanho da amostra dessa etapa, foi utilizada a equação citada por Mattar (1996) para uma população finita.

$$
n=\frac{N Z^{2} P Q}{\left(E^{2} N+Z^{2} P Q\right)}
$$

Onde:

n: tamanho da amostra;

$\mathrm{N}$ : tamanho da população;

P: proporção de ocorrência da variável em estudo na população $(0,5)$;

Q: proporção de não ocorrência da variável em estudo na população $(\mathrm{Q}=1$ - $\mathrm{P})$;

E: erro máximo admitido $(0,05)$;

Z: valor padrão determinado pelo nível de confiança.

Para o nível de confiança de $95 \%, Z=2$.

Portanto:

$\mathrm{n}=300 \cdot 2^{2} \cdot(0,5 \cdot 1 \cdot 0,5) /\left(0,05^{2} \cdot 250+2^{2}\right) \cdot(0,5 \cdot 1 \cdot 0,5)$

$\mathrm{n}=1200.0,25 / 0,0025.254 .0,25$

$\mathrm{n}=300 / 0,625+1$

$\mathrm{n}=300 / 1,625$

$\mathrm{n}=184,61$

O resultado do tamanho mínimo da amostra foi de 184,61, que foi arredondado: 185 questionários. A margem de erro perante o calculo é de 5\%.

O instrumento utilizado para obtenção de dados foi um questionário com base no Modelo Kano, de Tontini (2003), e a Matriz de Importância x Desempenho, proposto por Martilla e James (1977), buscando explorar a satisfação dos clientes do mercado União com atributos que relacionam produtos e serviços ofertados pela organização. Para tanto, foram analisados 18 atributos, conforme Quadro 1. 
Análise de prioridades de melhoria em relação à satisfação dos clientes de um mercado por meio de dois modelos de mensuração

Quadro 1 - Atributos utilizados na pesquisa da satisfação dos clientes.

\begin{tabular}{|l|l|}
\hline \multicolumn{2}{|l|}{ Atributos } \\
\hline Apresentação dos funcionários & Variedade de produtos \\
\hline Gentileza e cortesia no atendimento & $\begin{array}{l}\text { Organização e facilidade de encontrar os produtos nas } \\
\text { gôndolas }\end{array}$ \\
\hline Horário de funcionamento adequado & Exatidão do preço da gôndola com o preço do caixa \\
\hline Rapidez no atendimento no caixa & Condições de pagamento \\
\hline Atendimento na padaria & Promoções \\
\hline Qualidade dos produtos da padaria & Recebimento de promoções via Whatsapp \\
\hline Atendimento no açougue & Solução de problemas \\
\hline Qualidade dos produtos do açougue & Entrega a domicilio \\
\hline Diversidade e conservação de frutas e verduras & Limpeza e higiene do espaço \\
\hline
\end{tabular}

O questionário foi aplicado pessoalmente pelos pesquisadores na própria empresa, destinado a uma população diversa no que diz respeito à faixa etária, ao estado civil e ao sexo de cada um dos entrevistados. Ele foi dividido em cinco etapas: na primeira, mediu-se a satisfação atual dos clientes perante cada atributo com a organização, demonstrando o grau de satisfação existente em relação ao nível de desempenho atual encontrado em cada atributo.

A avaliação foi feita a partir de uma escala que varia de extremamente satisfeito $(+5)$ a extremamente insatisfeito (-5). O cliente indicou sua satisfação ou insatisfação atual referente às situações mencionadas, como mostra o Quadro 2.

Quadro 2 - Questões para identificação dos atributos pelo Modelo Kano.

\begin{tabular}{|l|l|l|l|l|l|l|l|l|l|l|l|l|}
\hline $\begin{array}{l}\text { Aponte o grau de desempenho, ou seja, sua SATISFAÇÃO } \\
\text { ATUAL em relação a cada atributo: }\end{array}$ & \multicolumn{6}{l|}{$\begin{array}{l}\text { Extremamente } \\
\text { Extremamente } \\
\text { Insatisfeito } \\
\text { Satisfeito }\end{array}$} \\
\hline 1.0 & Apresentação dos funcionários (uniformes) & -5 & -4 & -3 & -2 & -1 & 0 & 1 & 2 & 3 & 4 & 5 \\
\hline 2.0 & Gentileza e cortesia no atendimento dos funcionários & -5 & -4 & -3 & -2 & -1 & 0 & 1 & 2 & 3 & 4 & 5 \\
\hline
\end{tabular}

Fonte: Adaptado de Tontini, G. (2003). "Como identificar atributos atrativos e obrigatórios para o consumidor.” Revista de Negócios, Blumenau, v.8.

$\mathrm{Na}$ segunda parte, identificou-se o grau de importância para cada um dos atributos do questionário voltado à organização, na qual o cliente atribui significância numa escala de importância de sem importância (1) a extremamente importante (5). Já no terceiro momento, mediu-se a satisfação geral atual para com a organização, onde, novamente, as escalas variam de -5 (extremamente insatisfeito) a +5 (extremamente satisfeito). Na quarta parte, foram identificados os atributos segundo o Modelo Kano, através de duas perguntas, descrevendo respectivamente uma situação de aumento e outra de diminuição no desempenho de cada um dos atributos apresentados aos respondentes, outra vez avaliando de extremamente insatisfeito $(-5)$ a extremamente satisfeito $(+5)$. Por fim, na última parte, pesquisaram-se dados pessoais dos respondentes, pontos fortes e fracos e sugestões de melhoria, por meio de perguntas objetivas e fechadas.

A análise foi feita por meio de correlação estatística usando o SPSS versão 22.0, pela geração de gráficos no Excel, além da utilização da lógica não linear do Modelo Kano, bem como do gráfico da Matriz de Importância x Desempenho. 


\section{Análise e resultados dos dados}

Esta sessão apresenta os dados divididos em três blocos: inicialmente, será apresentado o perfil dos respondentes; logo após, a análise conjunta da Matriz de Importância x Desempenho e o Modelo Kano, além das análises estatísticas multivariadas (correlação); por fim, foram demonstrados os pontos fortes, os pontos fracos e as sugestões de melhorias.

\section{Perfil dos respondentes}

Para iniciar as análises, foram caracterizados os respondentes por meio de quatro questões fechadas que estão relacionadas ao perfil dos clientes, iniciando pela Tabela 1.

Tabela 1 - Sexo dos respondentes.

\begin{tabular}{c|c|c}
\hline Sexo & Quantidade & Percentual \\
\hline Feminino & 108 & $58,38 \%$ \\
\hline Masculino & 77 & $41,62 \%$ \\
TOTAL & 185 & $100 \%$ \\
\hline
\end{tabular}

Percebe-se, quanto ao sexo, que a maioria dos respondentes é do sexo feminino, correspondendo a $58,38 \%$ do total, sendo que $41,62 \%$ são do masculino.

Em relação à faixa etária, os resultados são apresentados na Tabela 2.

Tabela 2 - Faixa etária dos respondentes.

\begin{tabular}{c|c|c|c|c|c}
\hline Faixa etária & Quantidade & Percentual & Faixa etária & Quantidade & Percentual \\
\hline Até 18 anos & 10 & $5,41 \%$ & 41 a 50 anos & 38 & $20,54 \%$ \\
\hline 19 a 25 anos & 33 & $17,84 \%$ & 51 a 60 anos & 20 & $10,81 \%$ \\
\hline 26 a 30 anos & 43 & $23,24 \%$ & 61 a 70 anos & 10 & $5,40 \%$ \\
\hline 31 a 40 anos & 30 & $16,22 \%$ & 71 a 80 anos & 01 & $0,54 \%$ \\
- & - & - & TOTAL & 185 & $100 \%$ \\
\hline
\end{tabular}

Por meio da Tabela 2, observa-se que há predominância de um público adulto, justificado pelo maior número de respondentes estarem na faixa etária entre 26 e 50 anos, que somados representam $60 \%$ do total.

O estado civil em que os respondentes se encontram é apresentado na Tabela 3.

Tabela 3 - Estado civil.

\begin{tabular}{c|c|c}
\hline Estado civil & Quantidade & Percentual \\
\hline Solteiro & 64 & $34,59 \%$ \\
\hline Casado & 90 & $48,65 \%$ \\
\hline Amasiado & 20 & $10,81 \%$ \\
\hline Divorciado & 09 & $4,86 \%$ \\
\hline Viúvo & 02 & $1,08 \%$ \\
TOTAL & 185 & $100 \%$ \\
\hline \multicolumn{2}{|r}{}
\end{tabular}

A partir da Tabela 3, nota-se que a maioria dos respondentes é casado, sendo que representam $48,65 \%$ do total.

Os dados referentes ao período em que os respondentes compram no mercado estão dispostos na Tabela 4. 
Análise de prioridades de melhoria em relação à satisfação dos clientes de um mercado por meio de dois modelos de mensuração

Tabela 4 - Período de compra.

\begin{tabular}{c|c|c}
\hline Período & Quantidade & Percentual \\
\hline 0 a 1 ano & 01 & $0,54 \%$ \\
\hline 1 a 3 anos & 20 & $10,81 \%$ \\
\hline 4 a 7 anos & 54 & $29,19 \%$ \\
\hline 8 ou mais anos & 110 & $59,46 \%$ \\
TOTAL & 185 & $100 \%$ \\
\hline
\end{tabular}

Observa-se, por meio da Tabela 4, que a maioria dos respondentes é antiga e compra no mercado há oito anos ou mais, representando $59,46 \%$ do total.

\section{Satisfação geral}

Em relação à satisfação geral, os resultados são apresentados na Tabela 5.

Tabela 5 - Satisfação geral.

\begin{tabular}{c|c}
\hline \multicolumn{2}{c}{ Satisfação Geral } \\
\hline Média & 4,14 \\
\hline Percentual de satisfação & 92,09 \\
\hline
\end{tabular}

Com base nos dados da Tabela 5, percebe-se que as médias apresentaram-se de forma positiva, sendo que a satisfação geral atinge um percentual de satisfação igual a 92,09\% de aprovação, representando um nível de percepção de qualidade e de satisfação alto em relação aos produtos e aos serviços prestados pela organização.

Discussões de análise comparativa: matriz de importância $\mathrm{x}$ desempenho e modelo kano de qualidade atrativa e obrigatória

Os resultados da aplicação dos questionários foram processados com o intuito de identificar as prioridades de melhoria pela Matriz de Importância x Desempenho e a classificação dos atributos segundo o Modelo Kano.

\section{Análise pela Matriz de Importância x Desempenho}

As Figuras 3 e 4 mostram a Matriz de Importância x Desempenho para os atributos estudados. O ponto chave na análise pela Matriz de Importância x Desempenho é a posição das linhas divisórias dos quadrantes.

Figura 3 - Matriz de Importância x Desempenho para atributos pesquisados. 


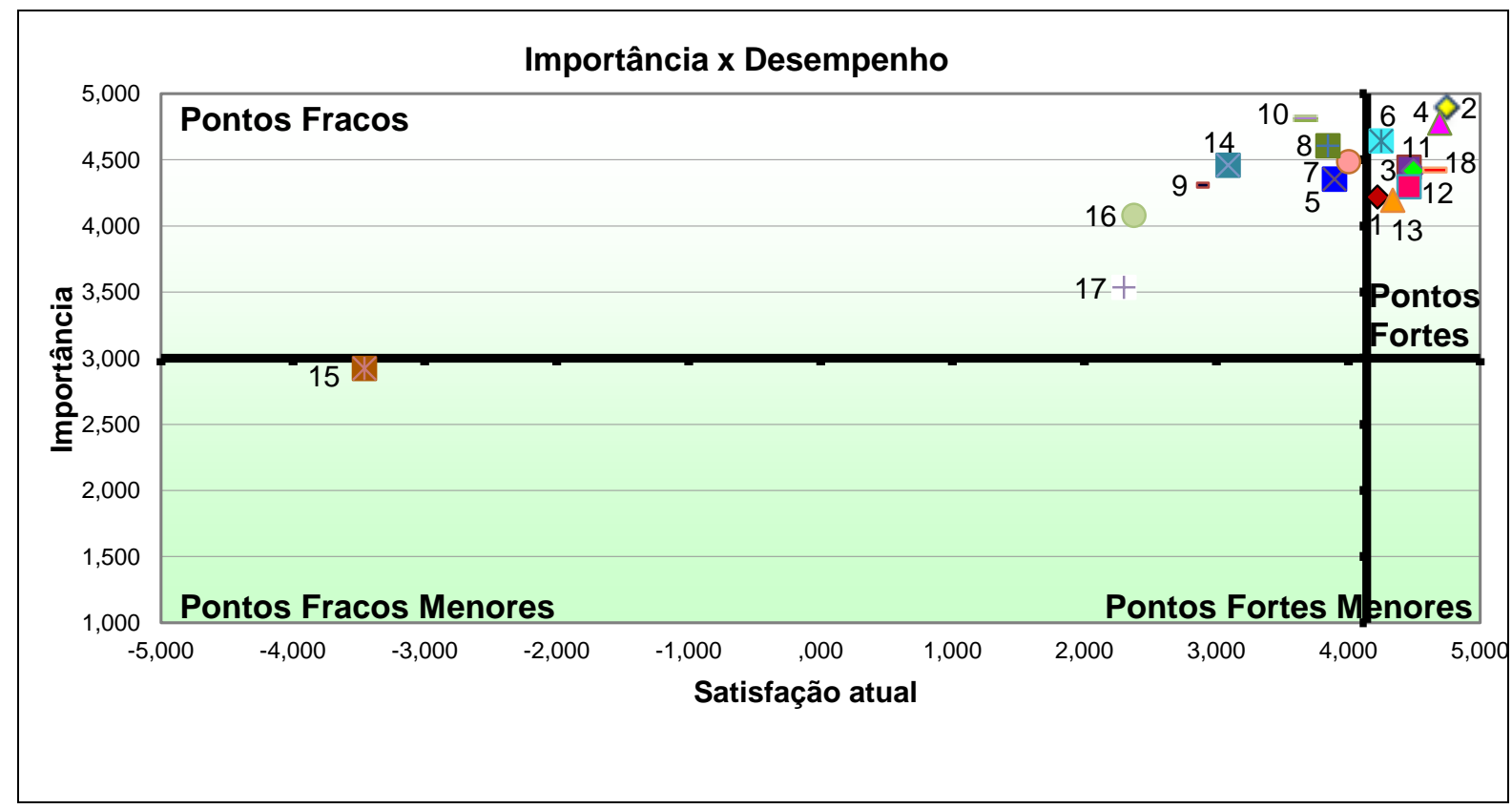

A Matriz da Figura 3 pode ser visualizada de forma ampliada (zoom) por meio da Figura 4.

Figura 4 - Ampliação da visualização da Matriz de Importância x Desempenho para atributos pesquisados.

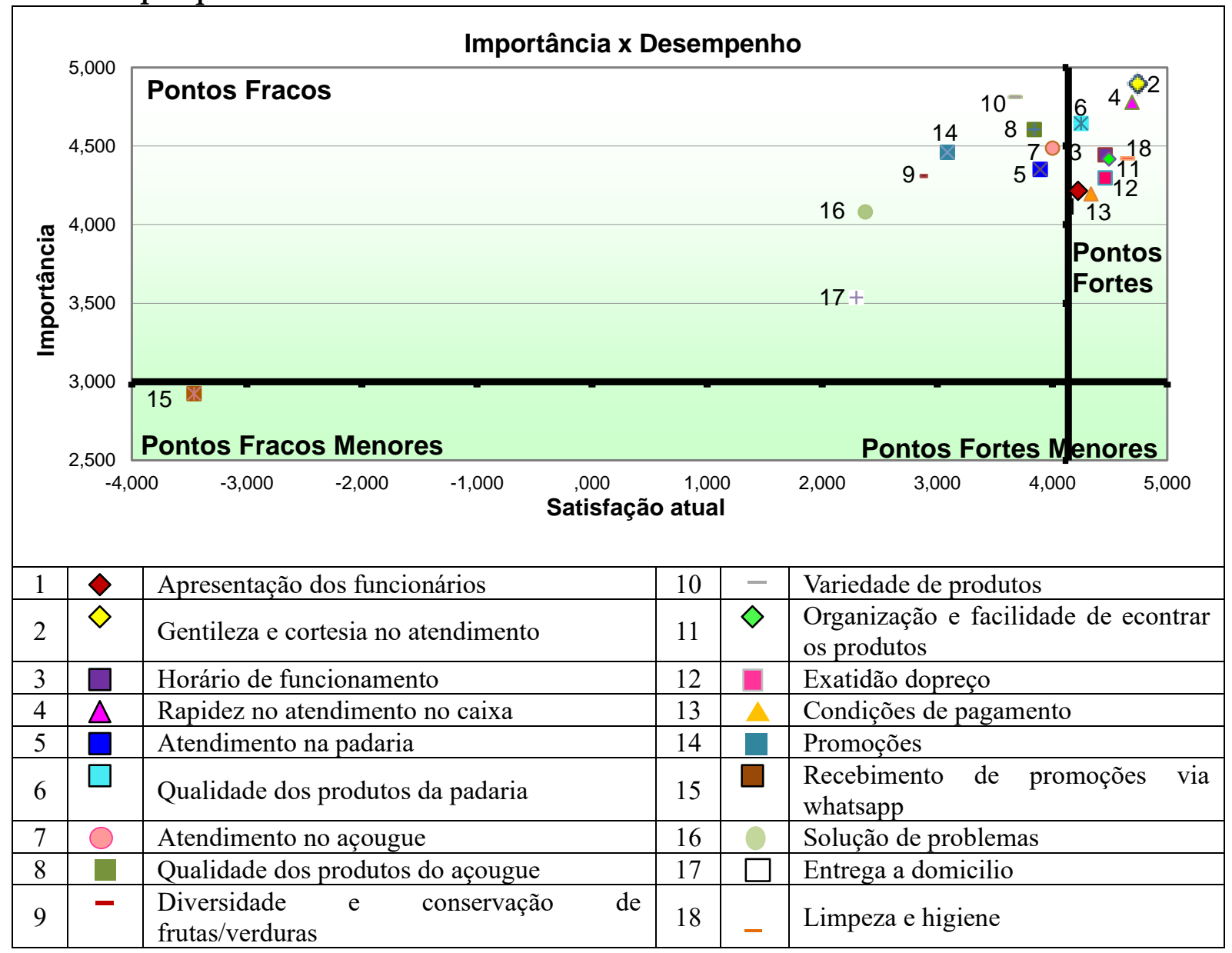


Análise de prioridades de melhoria em relação à satisfação dos clientes de um mercado por meio de dois modelos de mensuração

$\mathrm{Na}$ divisão dos quadrantes, foi utilizada uma das formas de divisão e análise de melhoria proposta por Matzler et al., (2004). A linha divisória de importância foi estabelecida em Importância (3), e a linha divisória de Satisfação Atual foi estabelecida pela média da Satisfação Geral Atual em relação aos atributos $(4,14)$.

No Quadrante I da Figura 4, observam-se a maioria dos atributos, nove no total, os pontos fortes da pesquisa: gentileza e cortesia no atendimento (2), rapidez no atendimento do caixa (4), qualidade dos produtos da padaria (6), horário de funcionamento (3), limpeza e higiene (18), organização e facilidade de encontrar os produtos nas gôndolas (11), exatidão do preço da gôndola com o preço do caixa (12), apresentação dos funcionários (1) e condições de pagamento (13).

No Quadrante II, variedade de produtos (10), promoções (14), qualidade dos produtos do açougue (8), atendimento no açougue (7), atendimento na padaria (5), diversidade e conservação de frutas e verduras (9), solução de problemas (16) e entrega a domicilio (17).

No Quadrante III da Figura 4, observa-se como ponto fraco menor um atributo: recebimento de promoções via Whatsapp (15).

No Quadrante IV, não há relação de atributos, o que significa que a empresa não está desperdiçando recursos.

\section{Análise pelo Modelo Kano de Qualidade Atrativa e Obrigatória}

A análise de dados segundo o Modelo Kano está representada nas Figuras 5 e 6 através do gráfico de dispersão do índice de satisfação do cliente. Nesse caso, a linha divisória dos quadrantes foi estabelecida em +1,00 para a escala do CS - Coeficiente de Satisfação, e -1,00 para o CI Coeficiente de Insatisfação.

Figura 5 - Gráfico de dispersão do índice de satisfação do cliente.

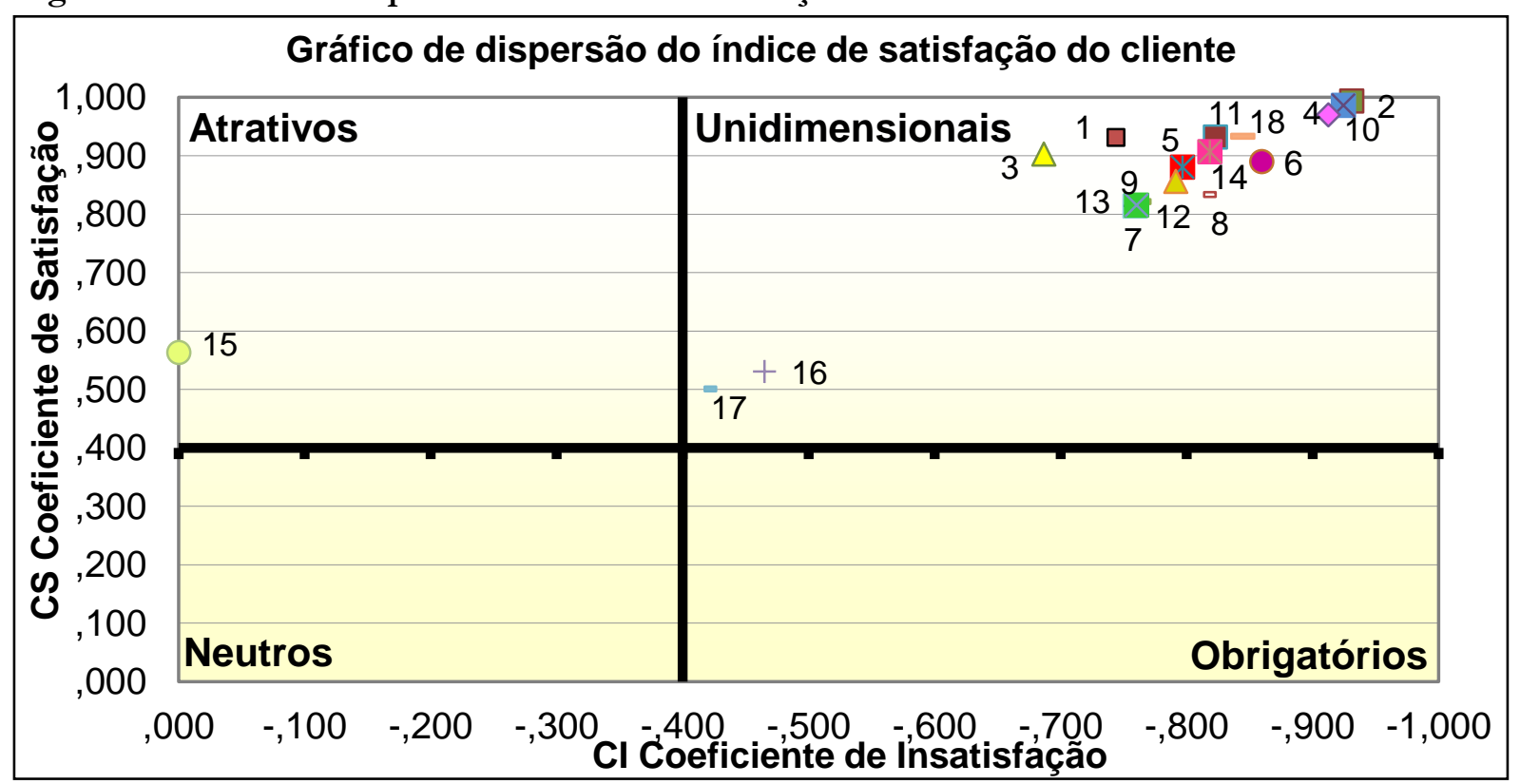

A Figura 5 pode ser visualizada de forma ampliada (zoom) por meio da Figura 6. 
Figura 6 - Ampliação da visualização do gráfico de dispersão do índice de satisfação do cliente.

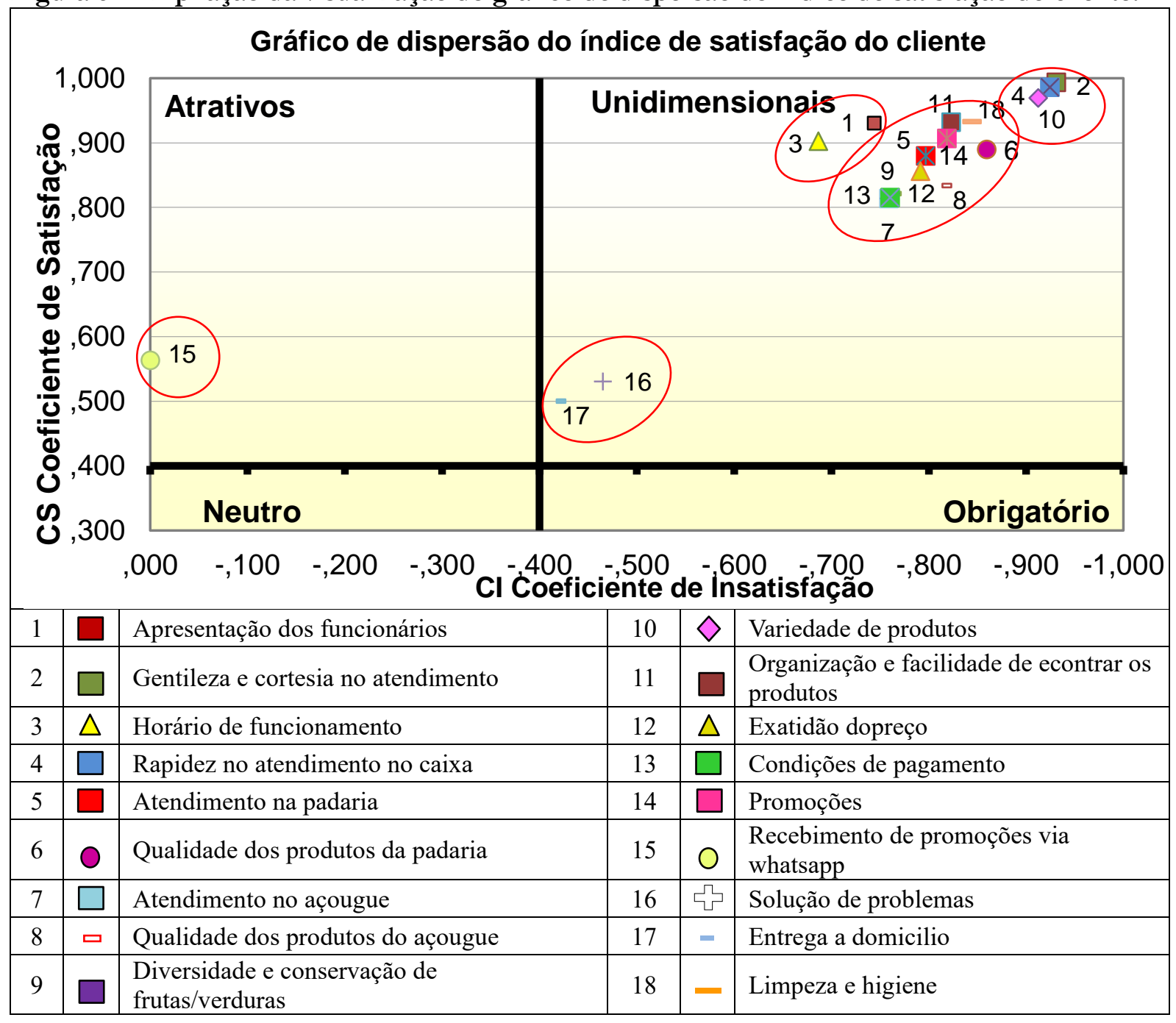

Por meio da Figura 6, identifica-se que os 17 atributos relacionados apresentam características de atributos unidimensionais, ou seja, a satisfação do cliente é proporcional ao nível de desempenho dos atributos. Os atributos foram demarcados com círculos que os diferem em grupos de acordo com sua relevância, na seguinte ordem: gentileza e cortesia no atendimento (2), rapidez no atendimento do caixa (4) e variedade de produtos (10) formam o primeiro grupo, seguidos de organização e facilidade de encontrar os produtos nas gondolas (11), limpeza e higiene do espaço (18), promoções (14), qualidade dos produtos da padaria (6), atendimento na padaria (5), exatidão do preço da gôndola com o preço do caixa (12), qualidade dos produtos do açougue (8), diversidade e conservação de frutas e verduras (9), condições de pagamento (13) e atendimento no açougue (7). No outro grupo, estão apresentação dos funcionários (1) e rapidez no atendimento (3). E, por fim, solução de problemas (16) e entrega a domicilio (17).

A Figura 6 revela ainda que há um atributo atrativo: recebimento de promoções via Whatsapp (15), no qual o desempenho é 0 . Isso se deve ao fato de que é um atributo não executado, no momento, pela organização.

Não houve incidência de atributos nos quadrantes neutros e obrigatórios, tornando-se itens sem avaliação para melhoria. 
Decisões de melhoria: Análise conjunta da Matriz de Importância $\times$ Desempenho e do Modelo Kano

Entende-se, de modo geral, que o consumidor tende a dar maior importância para atributos obrigatórios, decrescendo a importância para atributos unidimensionais, atrativos e neutros, respectivamente. Um desempenho superior em atributos obrigatórios não causa satisfação superior, a análise da Importância x Desempenho pode levar uma empresa a concentrar esforços em atributos cuja melhoria não trará grandes incrementos na satisfação geral. Já a baixa importância dada a atributos atrativos pode levar uma empresa a não melhorar atributos que poderiam trazer um diferencial em relação à concorrência, pois os mesmos geram uma satisfação superior, tratando-se de uma ação não esperada pelos consumidores como, por exemplo, o oferecimento de brindes.

Analisando pela conjunção a Matriz de Importância x Desempenho (Figura 4) e o Modelo Kano de Qualidade Atrativa e Obrigatória (Figura 6: no Quadrante II da Figura 4, observam-se 8 atributos, considerados os pontos fracos da pesquisa: variedades de produtos (10), promoções (14), qualidade dos produtos do açougue (8), atendimento no açougue (7), atendimento na padaria (5), diversidade e conservação de frutas e verduras (9), solução de problemas (16) e entrega a domicilio (17), que são os atributos considerados como prioridades de melhoria perante a organização, os quais deverão receber atenção imediata, já que, segundo a análise da Figura 6, os atributos são considerados unidimensionais, ou seja, quanto maior o nível de desempenho, maior será a satisfação do cliente, e vice-versa.

No Quadrante III da Figura 4, observa-se um atributo, considerado o ponto fraco menor da pesquisa, conforme informado na tabela pelo item: recebimento de promoções via Whatsapp (15), o qual pode receber atenção imediata buscando melhoria, já que, pela análise da Figura 6, o atributo é considerado atrativo. Ele é considerado ponto chave para a satisfação do cliente, e atendê-lo trará uma satisfação mais que proporcional, porém não traz insatisfação se não for atendido.

Concluindo a análise, baseando-se por meio de ambas as ferramentas, verifica-se que o mercado tem a oportunidade de avaliar e de buscar melhoria nos atributos descritos acima e que foram considerados de baixo desempenho de acordo com a opinião dos clientes, sendo os primeiros atributos a serem melhorados. Mas nada impede a empresa de, futuramente, analisar todo o conjunto de atributos, já que a grande maioria foi considerada unidimensional, baseando-se nesses para atingir um nível ainda maior de desempenho e garantir elevação no nível de satisfação geral.

\section{Análise de correlação pelo spss}

Outro método empregado para a análise da satisfação foi à análise de correlação por meio do Software SPSS (versão 22), que gerou a análise encontrada na Tabela 6.

O coeficiente de correlação de Spearman (1904) é uma medida de associação em que ambas as variáveis apresentam escala de mensuração pelo menos ordinal, na qual os elementos em estudo possam dispor-se em duas séries ordenadas. Trata-se de um teste não paramétrico, que determina $\mathrm{O}$ grau de associação entre as variáveis $\mathrm{X}$ e Y, objetivando estudar a correlação entre duas classificações (BAUER, 2007). 
Tabela 6 - Correlação de Spearman x Média da satisfação.

\begin{tabular}{l|c|c|c|c}
\hline Atributo & Correlação & Significância & $\begin{array}{c}\text { Satisfação } \\
\text { média }\end{array}$ & Ranking \\
\hline \multicolumn{3}{|c|}{ Abaixo da média } \\
\hline $\begin{array}{l}\text { Recebimento de promoções via Whatsapp } \\
\text { (15) }\end{array}$ &, $267^{* *}$ & Fraca & $-3,46$ & $1^{\circ}$ lugar \\
\hline Entrega a domicilio (17) &, 063 & Nula & 2,30 & $8^{\circ}$ lugar \\
\hline Solução de problemas (16) &, 087 & Nula & 2,37 & $9^{\circ}$ lugar \\
\hline $\begin{array}{l}\text { Diversidade e conservação de frutas e } \\
\text { verduras (9) }\end{array}$ &, $316^{* *}$ & Fraca & 2,85 & $2^{\circ}$ lugar \\
\hline Promoções (14) &, $254^{* *}$ & Fraca & 3,09 & $3^{\circ}$ lugar \\
\hline Variedade de produtos (10) &, $316^{* *}$ & Fraca & 3,68 & $4^{\circ}$ lugar \\
\hline Qualidade dos produtos do açougue (8) &, $200^{* *}$ & Nula & 3,89 & $5^{\circ}$ lugar \\
\hline Atendimento na padaria (5) &, $284^{* *}$ & Fraca & 3,90 & $6^{\circ}$ lugar \\
\hline $\begin{array}{l}\text { Organização e facilidade de encontrar os } \\
\text { produtos (11) }\end{array}$ &, $371^{* *}$ & ,371** & 4,00 & $7^{\circ}$ lugar \\
\hline \begin{tabular}{l|l|l|l|l} 
Condições de pagamento (13) \\
Limpeza e higiene do espaço (18)
\end{tabular} &, 006 & Nula & 4,76 & $\mathrm{I}^{\circ}$ lugar \\
\hline Horário de funcionamento (3) &, 124 & Nula & 4,69 & $\mathrm{H}^{\circ}$ lugar \\
\hline Atendimento no açougue (7) &, 136 & Nula & 4,65 & $\mathrm{G}^{\circ}$ lugar \\
\hline Qualidade dos produtos da padaria (6) &, $254^{* *}$ & Fraca & 4,46 & $\mathrm{~F}^{\circ}$ lugar \\
\hline Exatidão no preço (12) &, $234^{* *}$ & Fraca & 4,46 & $\mathrm{E}^{\circ}$ lugar \\
\hline Apresentação dos funcionários (1) &, $261^{* *}$ & Fraca & 4,34 & $\mathrm{D}^{\circ}$ lugar \\
\hline Gentileza e cortesia no atendimento (2) &, $278^{* *}$ & Fraca & 4,25 & $\mathrm{~B}^{\circ}$ lugar \\
\hline Rapidez no atendimento no caixa (4) &, $360^{* *}$ & Fraca & 4,22 & $\mathrm{~A}^{\circ}$ lugar \\
\hline Satisfação Geral (19) & 1,000 & & 4,14 & \\
\hline
\end{tabular}

A classificação de correlação de Spearman x Média de satisfação é classificada em 0,0 até $0,20 *$ correlações nulas; 0,21 até $0,40^{*}$ correlações fracas; 0,41 até $0,70^{*}$ correlações substanciais; 0,71 até 0,90* correlações fortes; e 0,91 até 1,0 correlações extremamente fortes (RODRIGUES, 2010).

De acordo com os dados analisados, existem 13 atributos que são de extrema importância para os clientes, os quais estão identificados na Tabela 6 com “**”, sendo eles: recebimento de promoções via Whatsapp (15), diversidade e conservação de frutas e verduras (9), promoções (14), variedade de produtos (10), qualidade dos produtos do açougue (8), atendimento na padaria (5), atendimento no açougue (7), qualidade dos produtos da padaria (6), exatidão do preço (12), apresentação dos funcionários (1), gentileza e cortesia no atendimento (2), rapidez no atendimento no caixa (4) e organização e facilidade de encontrar os produtos (11).

Dentre esses dados, 7 dos atributos apresentam uma satisfação média maior que a Satisfação Geral (Tabela 6), sendo assim, esses atributos são de extrema importância para os clientes, porém já estão satisfazendo-os, dessa forma, dispensam uma atenção imediata para melhoria.

Já os atributos que são menores que a satisfação geral e são elencados como importantes para os clientes devem ser analisados minuciosamente para melhoria imediata, pois, por serem de tal importância para os clientes e estarem abaixo da média relacionada, não estão atendendo às expectativas dos clientes. A listagem desses atributos inclui: recebimento de promoções via Whatsapp (15), diversidade e conservação de frutas e verduras (9), promoções (14), variedade de produtos (10), qualidade dos produtos do açougue (8), atendimento na padaria (5) e organização e facilidade de encontrar os produtos (11). O primeiro atributo citado representa algo que está em desuso e, portanto, segundo os clientes, é algo importante e merece voltar ao uso.

Foram constatados cinco tópicos sem correlação (sem importância) classificados com correlação nula, que devem ficar em último plano no delineamento de melhorias, sendo eles: entrega a domicilio (17), solução de problemas (16), condições de pagamento (13), limpeza e higiene do espaço (18) e horário de funcionamento (3). Os três últimos citados estão acima da satisfação geral, 
ou seja, pode haver um desperdício de recursos focalizados a esses atributos, que podem ser estagnados no momento.

Além disso, todos os tópicos devem ser avaliados pela empresa, buscando explorar melhoramento para atrair clientes novos e melhor satisfazer os clientes atuais.

\section{Pontos fortes, pontos fracos e sugestões de melhoria}

Solicitou-se aos respondentes que indicassem de forma descritiva pontos fortes em relação aos produtos e serviços prestados pela loja. Dos 185 respondentes, obtiveram-se 204 respostas de acordo com os pontos fortes descritos pelos clientes. O número de pontos fortes citados é maior que o de respondentes porque alguns dele apontaram dois pontos que acreditam ser positivos. Os dados obtidos são apresentados na Tabela 7.

Tabela 7: Pontos fortes indicados.

\begin{tabular}{l|c|l|c}
\hline \multicolumn{1}{c|}{ Pontos fortes } & \multicolumn{1}{c|}{ Citações } & \multicolumn{1}{c}{ Pontos fortes } & Citações \\
\hline Localização & 41 & Opções de pagamento & 06 \\
\hline Atendimento & 38 & Açougue & 06 \\
\hline Padaria & 28 & Entrega a domicilio & 06 \\
\hline Preços acessíveis & 16 & Promoções & 06 \\
\hline Variedade perante a concorrência & 11 & Atendimento via telefone & 04 \\
\hline Açougue - peixes & 10 & Variedade de produtos coloniais & 04 \\
\hline Sorteio dos R\$50 & 08 & Organização & 04 \\
\hline Higiene & 06 & Confiança & 04 \\
\hline Qualidade dos produtos & 06 & & - \\
\hline
\end{tabular}

É visível uma grande satisfação dos clientes no que diz respeito à localização, ao atendimento, à padaria, aos preços acessíveis, à variedade de produtos perante a concorrência, e aos peixes disponíveis no açougue, sendo, respectivamente, os primeiros e mais apontados atributos.

Com oito citações, segue o item sorteio dos $\mathrm{R} \$ 50,00$, higiene, qualidade dos produtos, opções de pagamento, açougue, entrega a domicílio e promoções, ambos com seis citações, seguidos de atendimento via telefone, variedades de produtos coloniais, organização e confiança, que, citados quatro vezes cada, completam e finalizam a listagem.

Dos 185 respondentes, obtiveram-se 117 respostas sobre os pontos fracos. Os dados obtidos são apresentados na Tabela 8.

Tabela 8: Pontos fracos indicados.

\begin{tabular}{l|c|l|c}
\hline \multicolumn{1}{c|}{ Pontos fracos } & Citações & \multicolumn{1}{c|}{ Pontos fracos } & Citações \\
\hline Preços elevados & 24 & Diversidade de produtos na padaria & 09 \\
\hline Diversidade de produtos & 21 & Diversidade de frutas e verduras & 08 \\
\hline Estacionamento & 20 & Atendimento na padaria & 07 \\
\hline Poucas promoções & 15 & Carne dura no açougue & 03 \\
\hline Qualidade das frutas e verduras & 10 & & - \\
\hline
\end{tabular}

É possível identificar, por meio da Tabela 8, que os pontos fracos com maior percepção dos clientes são preços elevados, diversidade de produtos, estacionamento e poucas promoções.

Outros pontos fracos que também se mostraram significativos são qualidade de frutas e verduras, com dez citações; diversidade de produtos na padaria, com oito citações; atendimento na padaria e carne dura no açougue, com sete e três citações, respectivamente, complementando a listagem.

Dos 185 respondentes, obtiveram-se 119 respostas à questão de sugestões de melhoria. Os dados obtidos são apresentados na Tabela 9. 
Tabela 9 - Sugestões de melhoria.

\begin{tabular}{l|c|l|c}
\multicolumn{1}{c|}{ Sugestões } & Citações & \multicolumn{1}{c|}{ Sugestões } & Citações \\
\hline Diversificar/variar os produtos & 22 & $\begin{array}{l}\text { Melhorar qualidade e variedade de frutas } \\
\text { e verduras }\end{array}$ & 11 \\
\hline Mais promoções & 18 & Abrir ao meio dia & 10 \\
\hline Melhorar o estacionamento & 15 & Diversificar/inovar os produtos da padaria & 09 \\
\hline Diminuir o preço dos produtos & 14 & $\begin{array}{l}\text { Mais funcionários que disponibilizem } \\
\text { tempo ao cliente }\end{array}$ & 04 \\
\hline Abrir aos domingos meio período & 14 & Melhor exposição das carnes no açougue & 02 \\
\hline
\end{tabular}

Por meio da Tabela 9, observa-se que os clientes almejam algumas mudanças nos serviços e produtos oferecidos. As sugestões mais evidentes são diversificação e variação de produtos, maior número de promoções, melhoria do estacionamento, diminuir o preço dos produtos e abrir aos domingos meio período foram os mais citados; seguindo de melhorias na qualidade e variedade de frutas e verduras, mencionada 11 vezes; abrir ao meio dia, mencionada 10 vezes; diversificar/inovar os produtos da padaria, mencionada 9 vezes; mais funcionários que disponibilizem tempo ao cliente e melhor exposição das carnes no açougue, mencionadas 4 e 2 vezes, respectivamente.

\section{Considerações finais}

Este estudo objetivou a análise de oportunidades de melhoria em relação à satisfação dos clientes de um mercado através da análise conjunta da Matriz de Importância x Desempenho e do Modelo Kano de Qualidade Atrativa e Obrigatória. Os resultados demonstram a eficácia da análise conjunta por meio de dois métodos de mensuração.

Este trabalho não pretendeu esgotar a análise de todos os atributos importantes na satisfação dos clientes, apesar de contribuir na prática identificando prioridades de melhorias, mas principalmente mostrar a potencialidade de utilização conjunta do modelo kano de qualidade com a matriz de importância x desempenho na descoberta de oportunidades de melhoria.

Como discutido em Huisknoen e Pirttila (1998), Matzler et al. (2004) e Tontini e Silveira (2005), a relação não linear e assimétrica entre o desempenho dos atributos e a satisfação por eles proporcionada pode levar a decisões equivocadas quando se analisa apenas a matriz de importância $\mathrm{x}$ desempenho. De maneira geral, atributos atrativos podem ser priorizados, investindo-se recursos em atributos não críticos (apesar de gerarem satisfação, se melhorados) ou, por outro lado, atributos críticos podem ser despercebidos, quando a ausência de más experiências anteriores pode levar os clientes a classificá-los como pouco importantes (HUISKNOEN; PIRTTILA, 1998). Neste estudo, verificou-se que a utilização conjunta do modelo kano com a matriz de importância x desempenho melhorou a qualidade de decisão. Atributos que têm grande impacto na satisfação, se melhorados, foram descobertos e priorizados. Já atributos que seriam priorizados, se analisados apenas pela matriz de importância x desempenho, deixariam de ser considerados críticos por serem classificados como atrativos pelo modelo Kano, sendo uma opção da empresa melhorá-los ou não.

O trabalho utilizou o questionário do modelo Kano com respostas com escala ordinal adaptado de Tontini (2003), conforme Quadro 2. No questionário, o respondente indicou o seu grau de satisfação ou insatisfação com o aumento ou diminuição do desempenho dos diferentes atributos. Esse método pretende refinar a identificação dos atributos pelo modelo Kano, uma vez que procura identificar o impacto da variação do desempenho dos atributos na satisfação dos clientes e não apenas se o cliente fica ou não satisfeito com uma determinada situação. Por outro lado, essa forma de identificação da classificação dos atributos do modelo Kano ainda carece de estudos mais aprofundados para se descobrir a melhor maneira de aplicação.

Como estudos futuros, pretende-se estudar como os gaps entre a satisfação atual e a expectativa de satisfação com a melhoria do desempenho poderiam ser utilizados junto com o modelo kano e com a matriz de importância e desempenho para identificar oportunidades de melhoria. 
Análise de prioridades de melhoria em relação à satisfação dos clientes de um mercado por meio de

\section{Referências}

ALVES, A. C., RAMALHO, A. M. C., SOUSA, C. M. de, SILVA, S. S. F. da, LACERDA, C. de S. \& JÚNIOR, J. J. S. (2015). A qualidade do serviço e a satisfação do cliente. Revista Pensamento \& Realidade. 30(4), 46-61.

AMARAL, S, A; DANTAS, E, B. Satisfação de clientes, marketing e serviços de informação (2015).

ASSOCIAÇÃO BRASILEIRA DE SUPERMERCADOS, ABRAS. Economia e pesquisa. São Paulo, (2019).

BAUER, L. Estimação do Coeficiente de Correlação de Sperman Ponderado. Porto Alegre, 2007.

BERGER, C. et al. Kano's methods for understanding customer-defined quality. Center for Quality Management Journal, v.2, n.4, p.33-35, 1993.

BINOTTO, S., DENARDIN, É., MEDEIROS, F., BOLIGON, J., \& MACHADO, F. (2014). Os Fatores de Influência no Comportamento do Consumidor: um estudo em uma cooperativa agrícola do RS. Revista de Gestão e Organizações Cooperativas, 1(2), pp. 13-26.

COBRA, M. Administração de marketing no Brasil. 3ª ed. Rio de Janeiro: Elsevier, 2009.

COSTA, A. S. C.; SANTANA, L.C.; TRIGO, A.C. Qualidade do atendimento ao cliente: um grande diferencial competitivo para as organizações. Revista de Iniciação Científica - RIC Cairu, v.2, n. 2, p. 155-172, 2015.

CUMMINS, R.A.; GULLONE, E. Why we should not use 5-point Likert scales: the case for subjective quality of life measurement. In. International Conference on Quality of Life in Cities, 2., 2000, Singapore. Proceedings... Singapore, 2000.

FERNANDES, C.G. Aplicação da Matriz Importância x Desempenho de slack na análise de mercado em uma indústria de saneantes e domissanitários. Especialize Revista OnLine, IPOG, maio, 2012.

FIGUEIREDO, K.F.; OZÓRIO, G.B.; ARKADER, R. Estratégias de Recuperação de Serviço no Varejo e seu Impacto na Fidelização dos Clientes. Revista de Administração Contemporânea. v.6, n.3, p.55-73, Set./Dez., 2002.

FIGUEREDO, M,S. Percepções sobre os atributos de qualidade da Associação Educacional do Vale do Itajaí Mirim a partir da integração do Modelo Servqual e Kano. 2005. 165 f. Dissertação (Mestrado em Administração) - Centro de Ciências Sociais Aplicadas, Universidade Regional de Blumenau, Blumenau, 2005.

GIL, A. C. Como elaborar projetos de pesquisa. 4. Ed. São Paulo: Atlas, 2002.

GIL, A. C. Métodos e técnicas de pesquisa social. 5. Ed. São Paulo: Atlas, 1999.

HUISKNOEN, J.; PIRTTILA. T. Sharpening logistics customer servisse strategy planning by applying Kano's quality elemento classification. International Journal of Produvtion Economics, n.56/57, p.253-260, 1998.

JESUS, C,O, J; CARVALHO, C,F; OLIVEIRA, R,C,R; NETO, F,M,H; TELES, B, B. Análise da Satisfação dos clientes no setor de Serviços: Estudo de caso em uma Empresa distribuidora de alimentos na cidade de Nossa Senhora do Socorro - SE. $2^{\circ}$ Congresso de Gestão, Negócios e Tecnologia da Informação, 2019.

JURAN, J, M; DEFEO, J, A. Fundamentos da qualidade. 1. Ed. Nova Iorque: Bookman, 2015.

KANO, N. et al. Attractive quality and must-be quality. Hinshitsu, v.14, n.2, p.147-56, 1984.

KANUK, L, L; SCHIFFMAN, L, G. Comportamento do consumidor. Rio de Janeiro: Editora LTC, 2009.

KOTLER, P; ARMSTRONG, G. Princípios de Marketing. 9a ed. São Paulo: Pearson Education, 2003

KOTLER, P. Administração de marketing: a edição do novo milênio. São Paulo: Prentice Hall, 2000. 
KOTLER, P. Administração de Marketing. São Paulo: Editora Pearson, 2006.

MAGALDI, S.; CRESCITELLI, E. A importância dos serviços suplementares no setor de serviços: uma análise do nível de satisfação do cliente. Revista de Gestão, v. 15, n. 3, art. 6, p. 67-75, 2008.

MARCHETTI, R.; PRADO, P. H. M. Avaliação da satisfação do consumidor utilizando o método de equações estruturais: um modelo aplicado ao setor elétrico brasileiro. RAC, v. 8, n. 4, p. 9-32, 2004.

MARTILLA, J, A; JAMES, J, C. Importance-performance analysis. The journal of marketing, p. 77-79, 1977.

MATTAR, F. N. Pesquisa de marketing. São Paulo: Atlas, 1996.

MATZLER, K. et al. Customer satisfaction with alpine ski areas: the moderating effects of personal, situational, and product factors. Journal of Travel Research, maio, p.402-413, 2008.

MATZLER, K; BAILOM, F; HINTERHUBER, H, H; RENZL, B; PICHLER, J. The asymmetric relationship between attribute-level performance and overall customer satisfaction: a reconsideration of the importance-performance analysis. Industrial marketing Management. v. 33, p. 271-277, 2003.

MATZLER, K; HINTERHUBER, H; BAILOM, F; SAUERWEIN, E. How to delight your customers. Journal of Product \& Brand Management, v. 5, 2, p. 6-18, 1996.

MOUTELLA, C. Fidelização de clientes como diferencial competitivo. Agosto de, 2003.

OLIVEIRA, V,S; PELISSARI, A,S; COELHO, J,H, M. Indicadores de satisfação: um estudo junto aos clientes do supermercado sigma. Qualit@s Revista Eletrônica. V, 14. N, 2; 2013.

PAULA, L, S; SOUZA, ANTONIO C, B. Fidelização de clientes e marketing de relacionamento. Valença - RJ, 2016.

RAUPP, F, M; BEUREN, I, M. Metodologia da pesquisa aplicável às ciências sociais. In. BEUREN, Ilse Maria. (Org). Como elaborar trabalhos monográficos em contabilidade. 3.ed. São Paulo: Atlas, 2006.

RODRIGUES, W. C. Estatística Aplicada, 8a Edição, Revisada e Ampliada. (2010), p. 42-43.

SACHS, J. Fidelização do cliente: Top strategies for increasing Your Company's BottomLine. Passo Fundo: Babelcube, Inc, 2015.

SALIBY, P,E. O marketing de relacionamento: o novo marketing da nova era competitiva. RAE. Revista de Administração de Empresas, vol. 37, n. 3, jul-set. 1997.

SALOMÃO, I,F,C,S; ABACAR, M; ALIANTE, G. Satisfação acadêmica em estudantes do curso de graduação em Ensino Básico da Universidade Pedagógica - Delegação de Nampula. Revista Brasileira de Ensino Superior, V. 4, N. 1 (2018).

SAUERWEIN, E. et al. The Kano Model: how to delight your customers. International Working Seminar on Production Economics, v.1 of the IX, Austria, p.313-327, Feb., 1996.

SHETH, J. N.; MITTAL, B.; NEWMAN, B. I. Comportamento do cliente: indo além do comportamento do consumidor. São Paulo: Atlas, 2001.

SILVA, M.A. A qualidade dos serviços como fator de diferenciação no varejo supermercadista. XII SemeAd: empreendedorismo e inovação, São Paulo/SP, 2009.

SILVA, S; SARAIVA, M. A Gestão da Qualidade Total como diferencial competitivo na satisfação e fidelização de cliente. III Encontro de Tróia 2012 - Qualidade, Investigação e Desenvolvimento, Tróia, 8 Junho 2012.

SOUSA, F, S, F. Satisfação de Clientes - O Caso de Uma Empresa Industrial. Dissertação de Mestrado - Faculdade de Economia da Universidade de Coimbra, 2011.

SOLOMON, M, R,. O comportamento do consumidor: comprando, possuindo e sendo. 9. ed. Porto Alegre: Bookman, 2011.

TARIGO, R; SILVA, J, E, A; GANZER, P, P; GASPERIN, D; CHAIS, C; MALAFIA G, C; OLEA, P, M. Processo de Decisão de Compra do Consumidor: O Caso de Uma Empresa Varejista XVI mostra de iniciação científica, programa de pós-graduação em administração - UCS, 2016. 
TONTINI, G; OSTETTO, F, S, M; ARRUDA, H,F; PICCOLO, J,M; GUARANHA, J, B, M. Análise de oportunidades de melhoria em laboratórios fotográficos através da integração da matriz de importância $x$ desempenho com o Modelo Kano de qualidade. Revista de Negócios, Blumenau, v. 9, 3, p. 179-188, jul./set. 2004.

TONTINI, G., Como identificar atributos atrativos e obrigatórios para o consumidor. Revista de Negócios, Blumenau, v. 8, 2003.

TONTINI, G,; SILVEIRA, A. Identification of critical atributes of success in products and services: na alternative to importance - performance analysis. In: Business Association os Latin American Studies - Balas 2005, Madrid. Proceedings... Madrid, 2005, p.1-18.

TRIERWEILLER, A. C., PRUDÊNCIO, M. V., WEISE, A. D., PEIXE, B. C. S. \& BORNIA, A. C. Diagnóstico de satisfação dos clientes organizacionais: um estudo de caso na Sigma LTDA. Revista GEPROS, 2011.

VIEIRA, F,M. A importância do marketing de vendas para as organizações. Trabalho de Conclusão de Curso apresentado à Faculdade Pitágoras. Contagem, 2018.

WALTER, S. A., Antecedentes da satisfação e da lealdade de alunos de uma instituição de ensino superior. 2006. 167 f. Dissertação (Mestrado em Administração) - Centro de Ciências Sociais Aplicadas, Universidade Regional de Blumenau, Blumenau, 2006. 